\title{
Mini Workshop - Real World Engineering Projects: Discovery-Based Curriculum Modules for First-Year Students
}

\author{
Joan Carletta, Pamela Bhatti, Tom Hartley, Yanfei Liu, Andrea Mitofsky, James A. Smith, \\ Loren Wyard-Scott and Alfred Yu \\ carlett@uakron.edu, pamela.bhatti@ece.gatech.edu, thartley@uakron.edu, liu@engr.ipfw.edu, mitofskya@trine.edu, \\ jasmith@ee.ryerson.ca, wyard@ece.ualberta.ca, alfred.yu@hku.hk
}

\begin{abstract}
This mini workshop is organized to provide an interactive forum for the introduction a set of six new curriculum modules developed under IEEE's Real World Engineering Projects (RWEP) program. The modules, which are representative of a larger collection of curriculum modules available to the public via an open-access RWEP web portal, are designed for use in the first-year engineering and computer science classroom, and are hands-on, team-based projects that emphasize the societal impact of the work that engineers do. After a brief introduction to the RWEP program and the six showcased curriculum modules, the authors of the modules will present their ideas and demonstrate the laboratory activities associated with their modules in interactive, informal simultaneous sessions.
\end{abstract}

Index Terms - laboratory instruction, first-year students, curriculum development, engineering education.

\section{WORKSHOP GOALS AND EXPECTED OUTCOMES}

The IEEE RWEP program's ongoing goal is to develop an on-line, open-access library of high quality, hands-on, teambased curriculum modules for use in first-year college courses in electrical engineering (EE), computer engineering $(\mathrm{CE})$, biomedical engineering (BE), electrical engineering technology (EET), and computer science (CS). These curriculum modules are designed to be used by faculty members around the world who teach first-year students in introductory laboratory courses. The modules are designed as stand-alone units each covering about two weeks of instruction, so that faculty can pick and choose the modules they like to build a course that meets their needs. The curriculum modules are specifically designed to be discovery-based, and to illustrate real-world contemporary problems whose engineering solutions benefit society.

The RWEP approach to first-year instruction is expected to help with retention of students, by clearly demonstrating the relevance of fundamental engineering and science concepts to real-world, contemporary problems, and by showcasing the impact that engineering has on society. Students are guided in discovering the importance of a contemporary problem in a way that will excite their interest in creative solutions. The modules make use of activities that allow students to discover key concepts about engineering science and design in a hands-on way; they demonstrate how and why technical methods work, rather than simply providing a recipe for a solution. The societal impact focus of the modules is expected to help in the retention of all students, and particularly women, by providing the students with opportunities to understand how their own future work might help others.

The goal of this mini workshop is to showcase new curriculum materials that have been developed for first-year students under IEEE's Real World Engineering Projects (RWEP) program. The six curriculum modules being presented are representative of a larger set of modules available on-line via the RWEP portal, www.realworldengineering.org. The expected outcomes of the session are that attendees:

- Will be encouraged to use the curriculum modules in their own classrooms.

- Will engage in discussion about the importance of relaying to their students the benefit to society of what engineers do.

- Will exchange information on learning methods that successfully engage first-year students and support recruitment and retention of those students.

- Will be made aware of future opportunities to contribute to IEEE Educational Activities Programs curriculum development efforts, particularly through grants from future rounds of the RWEP program. This awareness will help the RWEP "gain momentum" in building and strengthening its library.

\section{MINI-WORKSHOP AGENDA}


The mini-workshop will begin with a 10 minute overview of the Real World Engineering Projects program, followed by a brief (10-minute) introduction to the six projects and a short question and answer session. The bulk of the miniworkshop (60 minutes) will be used for live project demonstrations by the authors of the curriculum modules, set up "poster-session style", i.e., with simultaneous, informal presentations by the six author presentations. Each project will have its own demonstration table, with posters of information and hands-on demonstrations and/or videos. This will allow for audience members to interact directly with the authors, and to try out the same sorts of activities that are proposed for the students. This will give audience members a strong sense of how the activities might work at their own institutions, and an opportunity to talk interactively with the authors about their own experiences with the project in the classroom.

\section{Highlighted RWEP Projects}

\section{The Coding of Sound by a Cochlear Prosthesis}

\section{Pamela Bhatti}

Cochlear prostheses provide functional hearing to severe to profoundly deaf individuals. By delivering electrical charge to the organ of hearing, the cochlea, such devices enable the excitation of auditory nerve elements. These elements conduct this information to the brain where it is ultimately perceived as sound. Residing external to the body is a microphone, speech processor, and wireless transmission link. These components extract, code, and transmit features of speech from sound waves. Implanted within the body are a receiver/stimulator, and an electrode array that delivers current through metal sites along its span. This enables targeting specific sound frequencies.

In this Real World Engineering Project, students learn about cochlear prostheses, and then synthesize a speech processor to mimic a cochlear prosthesis in the laboratory. By manipulating prewritten functional blocks of Matlab code, student teams process audio speech files to distill the spectral content into frequency channels, and extract the energy contained in each channel. The students then exercise the code to reconstruct speech signals and output audio files. Music also serves as an input for comparison with speech. In doing the exercise, students will gain an appreciation for the spectral content of sound, build a foundation for eventual study of filtering and signal reconstruction, and appreciate the impact that signal processing can have on people's lives.

\section{Human Energy Generation and Electrical Signal Measurement}

978-1-4244-6262-9/10/\$26.00 @2010 IEEE

\section{Tom Hartley}

Energy generation and its efficient usage is becoming a pervasive problem in today's society as fuel prices continue to increase. Providing low cost energy to remote areas, depressed regions, and third world countries would have a tremendous impact on their lifestyle. Also, many devices such as communications equipment have low power requirements, while many high power devices require high power for only short periods of time. In both cases, low cost, low power generators can provide enough stored energy to power devices and improve the quality of life for a substantial fraction of the world's population. Of particular importance to improved lifestyle is the pumping of water, purification of water, generation of light, and heating of food. Less significant, but also important are power tools, transportation, and communication. A clear understanding of these issues is important for the development of responsible engineers and citizens.

In this Real World Engineering Project, students experiment with the generation of power by a human source, using a bicycle attached to a DC generator. The energy that they harvest is stored in a portable battery pack with an on-board power inverter. They do hands-on experiments to determine how much power is needed to operate many of the devices in their own lives. They learn about the storage of energy in an electrical energy storage device, and determine the efficiency of the conversion of DC-energy into more usable AC-energy. In the process, they are introduced to the measurement of a variety of electrical signals, and also to an application of calculus fundamentals to energy and power.

\section{Energy Scavenging from Vibrations}

\section{Yanfei Liu}

Energy harvesting, or the process of acquiring energy from the surrounding environment, has been a persistent human endeavor throughout history, e.g., the use of watermills in ancient Greece, and sailboats by Phoenicians and Egyptians, circa 4000 B.C. These days there is an increasing interest in harvesting energy at much smaller scales for use in embedded systems. For these applications, the power requirements are of a very small scale, e.g., less than 100 $\mathrm{mW}$.

In this Real World Engineering Project, students design and build a system that harvests energy from vibrations. A vibrating system is built using Lego pieces; the amplitude and frequency of the vibrations are determined by the speed of the motor and by the method used to transfer the rotary motion of the motor shaft into a vibration. The device used to harvest energy from the vibrating system is a piezo buzzer; the signal from the piezo buzzer is rectified, and used to charge a battery. Students are guided in conducting several energy harvesting experiments. The goal is to design the vibrating system is to interface as efficiently as

\section{$40^{\text {th }}$ ASEE/IEEE Frontiers in Education Conference}

October 27 - 30, 2010, Washington, DC 
possible with the piezo buzzer, given its characteristics. In the background lecture, students are given a suggestion to use asymmetric cams as a possible way to generate vibrations. Students are, however, given freedom to use their creativity and try different methods and approaches.

\section{Language Identification Software}

\section{Andrea Mitofsky}

Spectral analysis is a fundamental technique for obtaining information about all types of signals. Spectral analysis has been used in particular to obtain information about a piece of work. For example, spectral analysis has been used to detect whether works, including audio files and pictures, have been tampered with. Spectral analysis has also been used to determine the author of a piece of literature, the artist of a painting, or the programmer of a piece of computer code. Most introductions to the spectral analysis involve Fourier transforms and require knowledge of calculus, making them inappropriate for use with first-year students. This project introduces spectral analysis at a firstyear level, requiring no advanced mathematics, and only limited programming.

In this Real World Engineering Project, students use spectral analysis to identify the language in which a text file is written, based on the frequencies of letters in the text. Students are given an example computer program which demonstrates reading from a file, extracting a character, and writing to another file. The students are then tasked with writing the part of the program that builds a histogram of letter frequencies. Students may also look at the frequencies of pairs of letters or of words. Computing frequencies of pairs of letters or of words requires more computation time than computing letter frequencies, and the code needed will be more complicated. However, the students will learn that the extra time and complexity provides more information that can be useful in language identification.

\section{Pico Power Generation for the Developing World}

\section{Loren Wyard-Scott and James Andrew Smith}

Access to electrical power in remote, undeveloped areas can be a barrier to socioeconomic development. If a household has access to enough power to provide even a single light in their residence, socioeconomic conditions are dramatically improved. The level of education in these households is often increased since residents are able to follow scholarly pursuits even after dark. While connecting a household to the electrical power grid is not an option for much of the developing world, an exciting and cost-effective approach to providing households with power is pico power generation. In these installations, low-power $(<5 \mathrm{~kW})$ generation methods are used to provide off-grid power. Although not sufficient to meet a Western-world consumer's demands, these installations are enough to provide people of the developing world with new options that improve quality of life. The solutions discussed and developed here are consistent with the growing need for energy- and environmentally-efficient designs as the world's growing population taxes our limited resources.

In this Real World Engineering Project, students are asked to design and construct a pico power generation facility that can artificially extend daylight hours in each household of a remote village by two hours every day. To help the students achieve these objectives, several topics are covered: power (theory and power consumption trends), generation methods (electromechanical and semiconductor methods), energy storage techniques, and hybrid solutions. Student teams will explore individual household, distributed community and centralized community pico power designs, which offer tradeoffs in cost, efficiency, complexity and convenience. Starting with structured laboratory exercises, students will show mastery in practical measurement techniques and have an opportunity to systematically experiment with many of the topics relevant to pico power generation. Provided with a set of reasonably scaled requirements and appropriate guidance, the students are asked to design, construct, test, and document a full pico power generation system.

\section{Electrocardiogram Amplifier Design Using Basic Electronic Parts}

\section{Alfred Yu}

Electrocardiograms reveal important information about heart health. The proper design of circuits for biomedical instrumentation can play an important role in measuring bio-potentials and assist in medical diagnoses accordingly.

In this Real World Engineering Project, students have a hands-on opportunity to develop an electrocardiogram (ECG) amplifier circuit from scratch, and thereby learn more about the technical details of bio-potential measurement devices. The students develop a three-lead ECG amplifier on a breadboard using basic electronic parts such as op-amp chips, resistors, and capacitors. They learn how to reduce power-line interference in ECG measurements, and learn about the dependence of detected ECG magnitude on the angle between a measurement lead and the actual direction of ECG potential. In the process, students gain an appreciation for the practical importance and technical details of amplifier circuits used for ECG potential measurements.

\section{AUTHOR INFORMATION}

Joan Carletta, Department of Electrical and Computer Engineering, The University of Akron, carlett@uakron.edu. 
Pamela Bhatti, School of ECE, Georgia Institute of Technology,pamela.bhatti@ece.gatech.edu.

Tom Hartley, Department of Electrical and Computer Engineering, University of Akron, thartley@uakron.edu.

Yanfei Liu, Electrical \& Computer Engineering, Indiana Purdue University Fort Wayne, liu@engr.ipfw.edu.

Andrea Mitofsky, Department of Electrical \& Computer Engineering, Trine University, mitofskya@trine.edu

James A. Smith, Department of Electrical and Computer Engineering, Ryerson University, jasmith@ee.ryerson.ca.

Loren Wyard-Scott, Department of Electrical and Computer Engineering, University of Alberta, wyard@ece.ualberta.ca.

Alfred Yu, Department of Electrical and Electronic Engineering, University of Hong Kong, Hong Kong, Alfred.yu@hku.hk. 